\title{
Evaluation of Peach Quality Attribute Using an Electronic Nose
}

\author{
Hongmei Zhang and Jun Wang* \\ Department of Biosystems Engineering, Zhejiang University, \\ 268 Kaixuan Road, Hangzhou 310029, P. R. China
}

(Received December 10, 2008; accepted July 10, 2009)

Key words: peach, quality, artificial neural networks, electronic nose, prediction

In this study, responses of a sensor array were examined to establish a quality index model for evaluating peach quality index. The results showed that the multiple linear regression model is effective for predicting quality index, with high correlation coefficients $\left(R^{2}=0.87\right.$ for compression force; $R^{2}=0.79$ for sugar content; $R^{2}=0.81$ for $\mathrm{pH}$ ) and relatively low average percentage errors $(9.66 \%, 7.68 \%$ and $3.6 \%$, for compression force, sugar content and $\mathrm{pH}$, respectively). The feed-forward neural network also provides an accurate quality index model with high correlations $\left(R^{2}=0.90,0.81\right.$ and 0.87 for compression force, sugar content and $\mathrm{pH}$, respectively) between predicted and measured values and relatively low average percentage errors $(6.39 \%, 6.21 \%$ and $3.13 \%$ for compression force, sugar content and $\mathrm{pH}$, respectively) for prediction. These results prove that the electronic nose has the potential to become a reliable instrument to assess fruit quality index.

\section{Introduction}

Monitoring and controlling quality is becoming a very important issue in the fruit industry since the state of ripeness during harvest, storage and market distribution determines the quality of the final product measured in terms of customer satisfaction. ${ }^{(1)}$ Currently, general soluble solids, acidity, flesh firmness or pressure testing and fruit surface ground color are used to determine fruit quality.(2) Most of these methods require the destruction of the samples used for analysis. This is why, nowadays, determination of ripeness and optimal harvest dates and predictions of storage life are mainly based on practical experience. Thus, there is a need to develop a nondestructive technique to evaluate quality index.

Peach aroma, a potential indicator of the physiological condition of the fruit, can be used to develop a consistent and reproducible nondestructive technique to evaluate peach quality from harvest to consumers. Several researchers have used gas chromatography and mass spectrometry to identify volatiles emitted during the maturation of peaches. The importance of studying these compounds comes also from the fact that, in many

*Corresponding author: e-mail: jwang@zju.edu.cn 
cases, a clear relationship has been found between aging and the quantity of some emitted volatiles such as linalool or benzaldehyde. ${ }^{(3)}$

An electronic nose system is a sensor-based technology, which considers the total headspace volatiles and creates a unique smell print. An electronic nose does not resolve a sample's volatiles into individual components, but responds to the whole set of volatiles in a unique digital pattern. These patterns are the signature of a particular set of aromatic compounds. For each process or application of interest, a database of such digitized patterns is created, called the training set. When an unknown sample is exposed to electronic nose sensors, the electronic nose first digitizes the sample's volatiles, and then compares them with the existing training set. Electronic nose technology is being investigated to discriminate different quality fruits, and some experiments were performed for apples, ${ }^{(4-6)}$ pears and nectarines ${ }^{(7,8)}$ and oranges..$^{(9)}$ For example, Saevels et al. ${ }^{(10)}$ demonstrated that an electronic nose with quartz microbalance sensors could be used to evaluate the optimal harvest date for Janagold and Braeburn apples. Gomez et $a l^{(11)}$ found that electronic nose technology using metal oxide sensors can predict storage shelf-life for mandarins. Brezmes et al. ${ }^{(1)}$ found that the olfactory system is able to classify fruit samples into three different states of ripeness (green, ripe and overripe) with very good accuracy for peaches and pears with a success rate above $92 \%$.

In the study of Pathange et al., ${ }^{(2)}$ maturity indices such as starch index and puncture strength were used to categorize Gala apples into three maturity groups referred to as immature, mature and overmature fruits. From discriminant analysis results, an electronic nose could effectively categorize Gala apples into the three maturity groups with a correct classification percentage of $83 \%$. Brezmes et al.(12) found that electronic nose signals correlate with classical fruit quality parameters such as firmness, starch index and acidity.

Pattern recognition routines based on either statistical methods or on an artificial neural network (ANN) are used to evaluate the responses of a sensor array.(13)

In the study of Jensen et al., ${ }^{(14)}$ the applicability of predicting quality changes during storage of pork scratchings, peanuts, oatmeal and muesli by a sensor array was investigated. The results clearly show that the sensor array could predict the concentration of volatiles with an acceptable accuracy. Rodriguez-Nogales and coworkers $^{(15-16)}$ applied multivariate regression techniques to predict the percentages of cow's, ewe's and goat's milk in a mixture and predict the bovine, ovine and caprine milk percentages in Panela cheese. These techniques were effective for the prediction of percentages of milk. However, it was found that multivariate linear regression led to more precise predictions than the other multivariate calibration methods. Pene et al. ${ }^{(17)}$ applied multivariate regression techniques to predict the percentage of hazelnut oil used to adulterate olive oil. Good values were obtained in both techniques for the parameters employed (standard errors of prediction (SEP) and prediction residual error sum of squares (PRESS)) to evaluate the effectiveness of the techniques. With the proposed method, minimum adulteration levels of $7 \%$ and $15 \%$ can be detected in refined and virgin olive oils, respectively. Jha et al. ${ }^{(18)}$ applied multivariate regression techniques to fit the maturity index and $L, a$ and $b$ values of 160 mango samples. The partial least-square and principal component regression models using the same variables 
could not produce a better performance than the multivariate linear regression (MLR) model. These statistical methods have been applied to the determination of firmness and yellowness of mangos during growth and storage ${ }^{(19)}$ and to the prediction of acidity, soluble solids and firmness of Satsuma mandarins. ${ }^{(20)}$

The objectives of this research were (1) to evaluate the potential of an electronic nose to predict the sugar content (SC), $\mathrm{pH}$ and firmness of peaches through comparison with standard destructive techniques to assess maturity characteristics and (2) to obtain the prediction models for each peach quality index.

\section{Materials and Methods}

\subsection{Materials}

The test material "Dabai" peaches were obtained from the "Niannian Mountain" orchard. The orchard is located in Yuhang, in the southeast of China. Peaches were picked on June 9, 16 and 23, 2006. Upon arrival at the laboratory, the peaches were inspected to ensure that they were uniform, undamaged and not infected with worms. Ninety peaches were used for the experiment (30 peaches for each picking date). All peaches were evaluated on the day they were picked.

\subsection{Sensor array and experimental setup}

The electronic nose consisted of a sensor array and a data acquisition card (NI USB-6009, National Instruments Corporation, USA). Eight commercial metal oxide sensors (6 TGS, Japanese manufacturer Figaro; MQ-3 and MQ-7 of metal oxide sensors, HANWEI Electronics Co., Ltd., Henan, China) were placed in a cycloidal chamber. Table 1 shows a list of all the sensors used and their main applications. This table contains currently known or specified reactions. To reach the working temperature (about $305^{\circ} \mathrm{C}$ ), the sensors were heated by applying a $5 \mathrm{~V} \mathrm{DC}$ voltage. The experimental setup is shown in Fig. 1. The response of the eight gas sensors was monitored using a data acquisition card (NI USB-6009) connected to a personal computer. With a pump, the gaseous compounds were sucked through the sensor array (flow rate $100 \mathrm{ml} / \mathrm{min}$ ). The

Table 1

Array components, main applications and detection ranges.

\begin{tabular}{lcc}
\hline \multicolumn{1}{c}{ Name } & Main application & Typical detection range (ppm) \\
\hline MQ-3 (S1) & Ethanol & $10-300$ \\
TGS822 (S2) & Solvent vapor detection & $50-5,000$ (Organic solvents) \\
MQ-7 (S3) & Carbon Monoxide & 20-2,000 \\
TGS800 (S4) & Air quality control & 1-10 (Cigarette smoke, gasoline exhaust...) \\
TGS824 (S5) & Toxic gas detection & 30-300 (Ammonia) \\
TGS813 (S6) & Combustible gas & detection \\
TGS880 (S7) & Cooking control & (Volatile gases and water vapour from food) \\
TGS825 (S8) & Hydrogen Sulphide & 5-100 \\
\hline
\end{tabular}




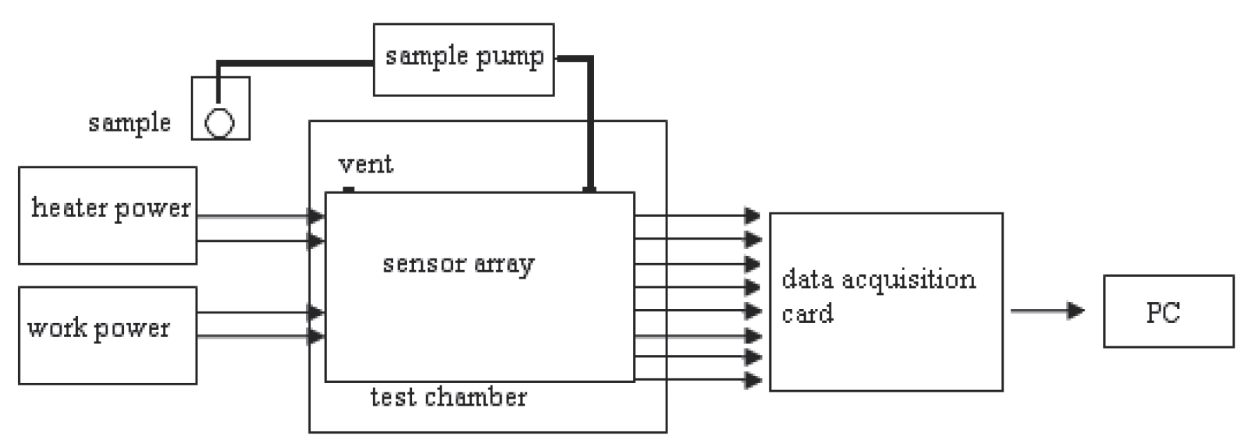

Fig. 1. Experimental setup.

experimental data was displayed in real-time on the computer screen and stored as text files on the disk for processing.

\subsection{Measurement of quality indices}

In order to assess real peach quality parameters at different picking times, firmness (compression force), SC and $\mathrm{pH}$ were determined. The $\mathrm{SC}$ of juice for each fruit was measured with a saccharimeter (Saccharimeter WYT-J 0-32\%, Beijing, China). The pH of juice was measured using a pH meter (Mettler Toledo Delta 320, Switzerland). Fruit firmness was evaluated in terms of maximum compression force (CF). The maximum compression force of all individual fruits was measured at three positions at intervals of approximately $120^{\circ}$ along the equator, perpendicular to the stem-bottom axis. The measurements were carried out using a universal testing machine (Model 5543 Single Column, Instron Corporation, Canton, MA, USA). The tests were performed using parallel plates. The tests were conducted over a distance of $11 \mathrm{~mm}$ by puncturing a fruit to a depth of $10 \mathrm{~mm}$, and the loading rate of the crosshead was $20 \mathrm{~mm} / \mathrm{min}$. Firmness was the mean of the "maximum forces" at the three points.

\subsection{Procedures}

The total number of peaches used in experiments was 90 (30 peaches for each picking date). Each fruit was placed into an airtight $500 \mathrm{ml}$ glass jar. The glass jar was then closed and the headspace inside it was equilibrated for $0.5 \mathrm{~h}$. The experiment was started when the resistance of the gas sensors remained stable at a high value. A fan homogenized the air in the test chamber. A small amount of the headspace gas to be tested was introduced into the test chamber. Then, data acquisition was started. The measurement phase lasted $90 \mathrm{~s}$, a sufficient time for sensors to reach a stable value. When a measurement was completed, the clean phase was activated, in which clean air was introduced into the test chamber. The main purpose was to clean the test chamber and return sensors to their baseline. The clean phase lasted $120 \mathrm{~s}$. The electronic nose was used at a temperature of $25 \pm 1^{\circ} \mathrm{C}$ and $50-60 \% \mathrm{RH}$ during all experiments. 
After headspace evaluation, each peach was removed from the jar and weighed on an electronic balance. The maximum compression force of all individual fruits was measured at three positions at intervals of approximately $120^{\circ}$ along the equator, perpendicular to the stem-bottom axis. Each fruit was cut into pieces and juice was squeezed out to evaluate the $\mathrm{SC}$ and $\mathrm{pH}$. The $\mathrm{SC}$ of juice was measured using a saccharimeter. The $\mathrm{pH}$ of juice was measured using a $\mathrm{pH}$ meter.

\subsection{Data analysis}

Quality indices and electronic nose sensor responses were measured using 90 peaches (30 peaches for each picking date). The responses of the sensor array were used together with quality parameters in statistical analysis. Principal component analysis (PCA) and linear discriminant analysis (LDA) were applied to determine whether the chemical sensor array can extract sufficient relevant features used for monitoring the ripening process. MLR and ANN analyses were used to establish prediction models. The sample data (90 peaches collected on three picking dates) were separated randomly into two groups: One group for the training set used to develop the training models (60 peaches, 20 for each picking date) and the other group for the prediction set (30 peaches, 10 for each picking date).

PCA is a chemometric linear, unsupervised and pattern recognition technique used for analyzing, classifying and reducing the dimensionality of numerical datasets in a multivariate problem. ${ }^{(21,22)}$ LDA is one of the most widely used classification procedures. The method maximizes the variance between categories and minimizes the variance within categories. It looks for a sensible rule to discriminate between categories by forming linear functions of the data maximizing the ratio of the between-group sum of squares to the within-group sum of squares. ${ }^{(23,24)}$

MLR analysis is a common method used in quantitative analysis. Equations relating the dependent variable behavior to the descriptors are developed with the following form:

$$
Y_{i}=\beta_{0}+\beta_{i} \times X_{i} \quad(i=1,2,3, \cdots, N),(1)
$$

where $Y_{i}$ is a dependent variable; $\beta_{0}$ is the intercept and $\beta_{i}$ are the regression coefficients of the independent variable $X_{i}$; and $N$ is the number of independent variables. In the present work, a forward stepwise MLR procedure is applied. ${ }^{(25)}$

In order to search for the best relationship for predicting fruit quality indices from sensor signal values, the quality of the calibration model was quantified by the accuracy standard error of calibration (SEC), SEP and the correlation coefficient $\left(R^{2}\right)$ between the predicted and measured parameters. A good model should have a low SEP and a high correlation coefficient. SEC and SEP are defined as follows:

$$
S E C=\sqrt{\frac{1}{I_{\mathrm{c}}-1} \sum_{i=1}^{I_{\mathrm{c}}}\left(\hat{y}_{i}-y_{i}\right)^{2}}
$$




$$
S E P=\sqrt{\frac{1}{I_{\mathrm{P}}-1} \sum_{i=1}^{I_{\mathrm{P}}}\left(\hat{y}_{i}-y_{i}-\text { bias }\right)^{2}},
$$

where $\hat{y}_{i}$ is the predicted value of the $i$ th observation, $y_{i}$ is the measured value of the $i$ th observation, and $I_{\mathrm{P}}$ is the number of observations in the training set.

$$
\text { bias }=\frac{1}{I_{\mathrm{P}}} \sum_{i=1}^{I_{\mathrm{p}}}\left(\hat{y}_{i}-y_{i}\right)
$$

The performance of the training models for prediction of maturity indices was tested with three picking dates of samples. The artificial neural network (ANN) model is a powerful study system and can actualize high nonlinearity mapping between input and output, and it was confirmed that this model can be used for any continuous nonlinear curve. For comparison, variables of the selected model were then analyzed using ANN techniques to check for better predictability. ${ }^{(26)}$

\section{Results and Discussion}

\subsection{Quality indices}

The mean values of the quality index are presented in Table 2. Regarding peach ripeness, the SC (mean value) increased from 5.97 to 6.55 , an increase of $9.72 \%$ after 14 days. The $\mathrm{pH}$ of peaches increased during ripening from 4.64 to 5.10 , with a total increase of $9.91 \%$ during the growth period of 14 days. The CF of peaches decreased with time from 65.36 to 15.11 , with a total decrease of $76.88 \%$ during the growth period of 14 days. The peaches were picked on 23 June, which was optimum for eating, and on 16 June, which was optimum for transportation and storage, according to an expert's experience.

\subsection{Electronic nose response to peach aroma}

Figure 2 shows a typical response of eight sensors for peaches. The gas response is given by $R / R_{0}$, where $R$ and $R_{0}$ express the resistance of a sensor in clean air and in detecting gas, respectively. It was shown that after an initial period of low and stable conductivity (when only clean air crosses the measurement chamber), conductivity increases sharply and then stabilizes after a collection time of $45 \mathrm{~s}$. In this research, stable signals $(60 \mathrm{~s})$ of each sensor were used in the analysis of the electronic nose.

Table 2

Mean values (with standard deviation) of maturity indices for "Dabai" peaches.

\begin{tabular}{lccc}
\hline Picking day & CF $(\mathrm{N})$ & $\mathrm{pH}$ & $\mathrm{SC}(\%)$ \\
\hline $09 / 06 / 06$ & $65.36 \pm 9.34$ & $4.64 \pm 0.29$ & $5.97 \pm 0.20$ \\
$16 / 06 / 06$ & $30.24 \pm 5.05$ & $5.04 \pm 0.22$ & $6.35 \pm 0.76$ \\
$23 / 06 / 06$ & $15.11 \pm 7.61$ & $5.10 \pm 0.14$ & $6.55 \pm 0.92$ \\
\hline
\end{tabular}




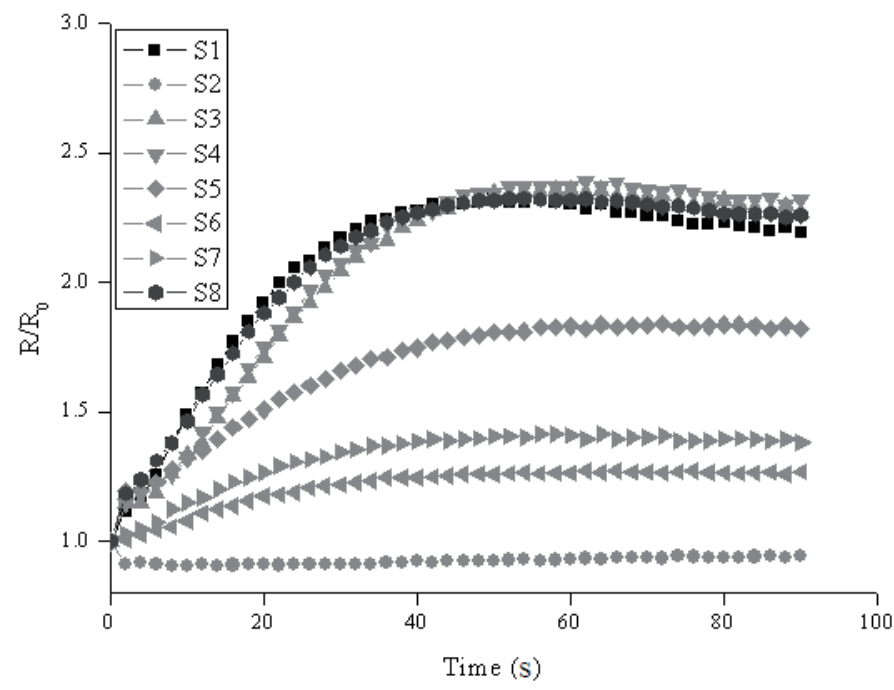

Fig. 2. Typical response curves of sensor array for peach headspace (picked on 23 June).

\subsection{Statistical analysis of electronic nose sensor response data}

In order to determine whether the chemical sensor array was able to distinguish between different picking dates, PCA and LDA were applied to the data obtained from 90 measurements using the olfactory system. By PCA, data from the samples picked on 9 June are clustered at the left of the plot, those from the samples picked on 23 June are located at the top right of the plot, and those from samples picked on 16 June are located at the bottom of the plot (Fig. 3(a)). After analyzing the same data set using LDA, the three groups (Fig. 3(b)) were more clearly distinguished than in the case of using PCA. In this plot, about $99 \%$ of the total variance of the data is shown. Functions 1 (LD1) and 2 (LD2) accounted for $76 \%$ and $23 \%$ of the variance, respectively. A clear evolution in the ripening process was observed. From the PCA correlation matrix, most of the signals of sensors were highly correlated $\left(R^{2}=0.95\right)$ to each other, whereas the correlation $\left(R^{2}\right)$ among a few signals of sensors was as low as 0.23. MANOVA indicated that the maturity groups were not all equal $(F=72, P<0.0001)$. Thus, it could be concluded that maturity affected electronic nose sensor responses, and the electronic nose was able to successfully distinguish the picking date of peaches.

\subsection{Multiple linear regression}

The MLR algorithm establishes the model that describes the relationship between sensor signals and fruit quality indices. In this analysis, the stepwise procedure of the statistical analysis system (SAS) was used. All variables used in the models are significant at the 0.01 level. The final set of selected equations was then tested for stability and validity by various statistical methods. The equation suitable for further 

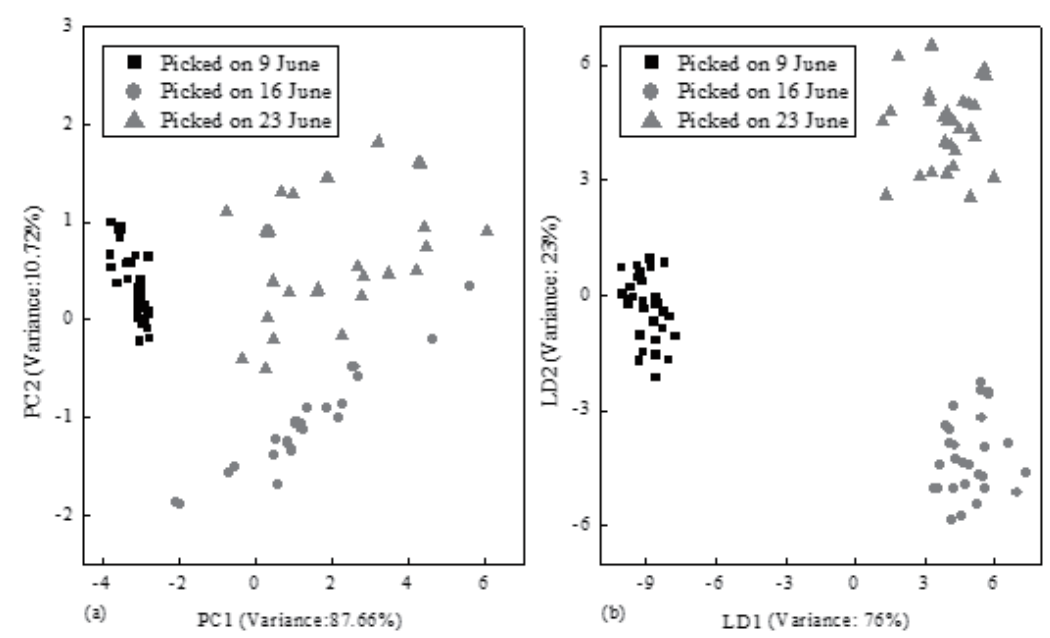

Fig. 3. Scatter plots of the first two main axes illustrating the E-nose sensor response data categorized into three groups: (a) PCA and (b) LDA.

consideration was chosen on the basis of four criteria, namely, $R^{2}$, SEP, $F$-values and the number of descriptors in the model. The best model, the MLR model, has high $R^{2}$ and $F$-values, low standard error prediction, least number of descriptors and high ability for prediction. The MLR algorithm establishes the model that describes the relationship between sensor signals and fruit quality index. The sample data ( 90 peaches from three picking dates) were separated randomly into two groups: one group for the calibration set was used to develop the calibration models (60 peaches, 20 samples each picking date) and the other group containing the remaining samples was used for the prediction set (30 peaches, 10 samples from each picking date). The predictive models for $\mathrm{CF}, \mathrm{SC}$ and $\mathrm{pH}$ thus developed are given below:

$$
C F=67.31-64.86 \times S 1+99.27 \times S 2+92.07 \times S 3-78.15 \times S 5-44.49 \times S 7 .
$$

$S 4, S 6$ and $S 8$ are not significant at the 0.01 level and are thus deleted from the model. $n=60 ; R^{2}=0.92 ; S E C=1.48 ; F=60.23 ; P<10^{-4}$,

$$
S C=-7.44+2.75 \times S 1-4.07 \times S 2+77.32 \times S 3-78.5 \times S 4+6.08 \times S 5+15.5 \times S 6-6.20 \times S 8 .
$$

$S 7$ is not significant at the 0.01 level and is thus deleted from the model. $n=60 ; R^{2}=0.82$; $S E C=0.27 ; F=39.26 ; P<10^{-4}$,

$$
p H=9.93-0.65 \times S 1-10.83 \times S 3+10.57 \times S 5-6.86 \times S 6+1.07 \times S 8 .
$$

$S 2$ and $S 7$ are not significant at the 0.01 level and are thus deleted from the model. $n=$ 
$60 ; R^{2}=0.88 ; S E C=0.15 ; F=21.55 ; P<10^{-4}$.

Here $n$ is the number of data points used in deriving the regression equation, $S i$ is the signal of the $i$ th sensor, $F$ is the F-test value of significance and $P$ is the significance level of the equation.

Large $R$ and $F$ values indicate an adequate fit. $\mathrm{CF}, \mathrm{SC}$ and $\mathrm{pH}$ values calculated on the basis of the above relationship are shown in Table 3 and compared with the experimental values. Figures 4-6 show the prediction ability of the electronic nose, where each square represents the predicted values versus the real value of each measurement. The figures illustrate a linear correlation between the responses of sensors and quality indices, indicating that the responses of sensors linearly correlated with $\mathrm{CF}$, $\mathrm{SC}$ and $\mathrm{pH}$. The measured $\mathrm{CF}$ values have a horizontal dispersion in relation to the diagonal line while predicted values do not. This is particularly true for fruit picked on 9 June, while the fruit picked on the other dates have another pattern. Also for SC data, measured values are, in general, higher than predicted values. The correlations of the training model for $\mathrm{CF}, \mathrm{SC}$ and $\mathrm{pH}$ for peaches were high with a low SEC. When the models were used to predict the other group samples of peaches, prediction results were also high. The MLR models appeared to have a high ability of prediction. The results are shown in Table 3.

\subsection{ANN analysis}

The artificial neural network used was a three-layer back-propagation network, with a tan-sigmoid transfer function in the hidden layers and a log-sigmoid transfer function in the output layer. The log-sigmoid transfer function was chosen because its output range $(0-1)$ is perfect for learning Boolean output values. The training algorithm was the variable learning rate backpropagation (traingdx) algorithm available in MATLAB' s Neural Network Toolbox. It has also shown high performance in pattern recognition problems. There are $20 \times 3=60$ samples ( 20 for each picking date) in the training set, and $10 \times 3=30$ samples ( 10 for each picking date) in the test set. The architecture of the artificial neural network chosen was $N \times(2 N+1)$ three-layer backpropagation in accordance with Kolmogorov's theorem, where $N$ is the sum of input neurons and three maturity indices are the target output.

A standard procedure in backpropagation ANN analysis is the training of the network using a set of data (consisting of input variables and target output), that, by means of iterative minimization of the prediction error, allows optimization of the adjustable

Table 3

Results of prediction of fruit quality indices obtained on the basis of electronic nose signal (MLR model).

\begin{tabular}{ccccccc}
\hline Quality indices & \multicolumn{6}{c}{ MLR } \\
\hline \multicolumn{7}{c}{ Calibration } \\
\hline$R^{2}$ & SEC & ERR & $R^{2}$ & SEP & ERR \\
\hline CF & 0.92 & 1.48 & 3.2 & 0.87 & 2.95 & 9.66 \\
SC & 0.82 & 0.27 & 6.67 & 0.79 & 0.50 & 7.68 \\
pH & 0.88 & 0.15 & 2.08 & 0.81 & 0.38 & 3.6 \\
\hline
\end{tabular}



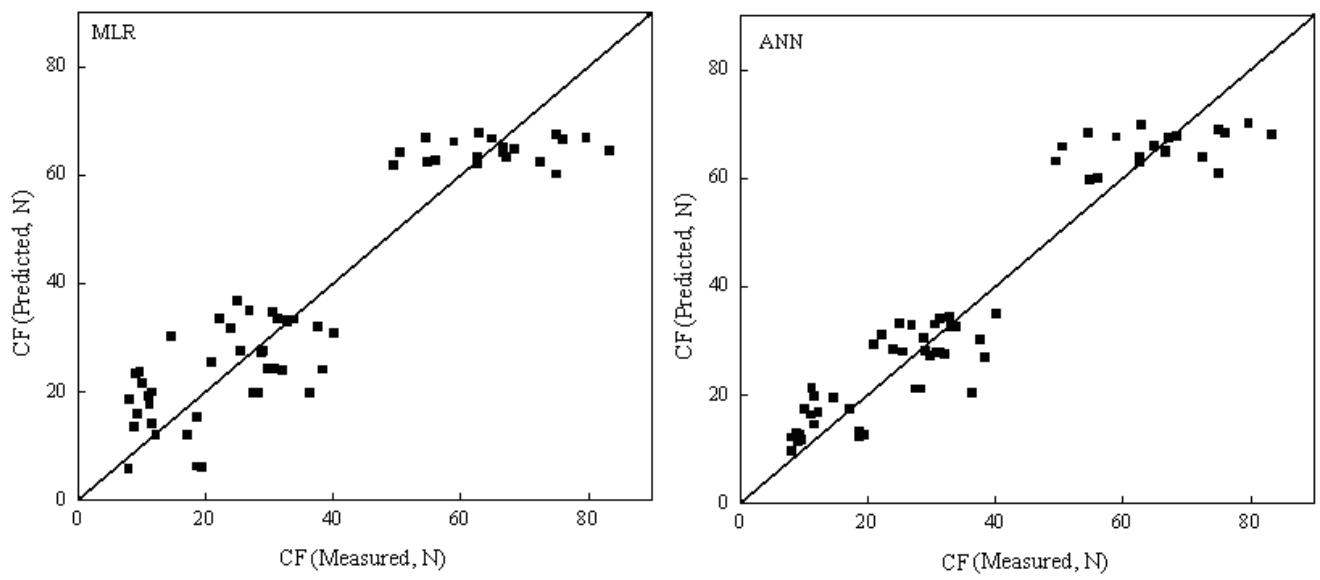

Fig. 4. Comparison of measured $C F$ values with predicted values obtained using ANN and MLR models.
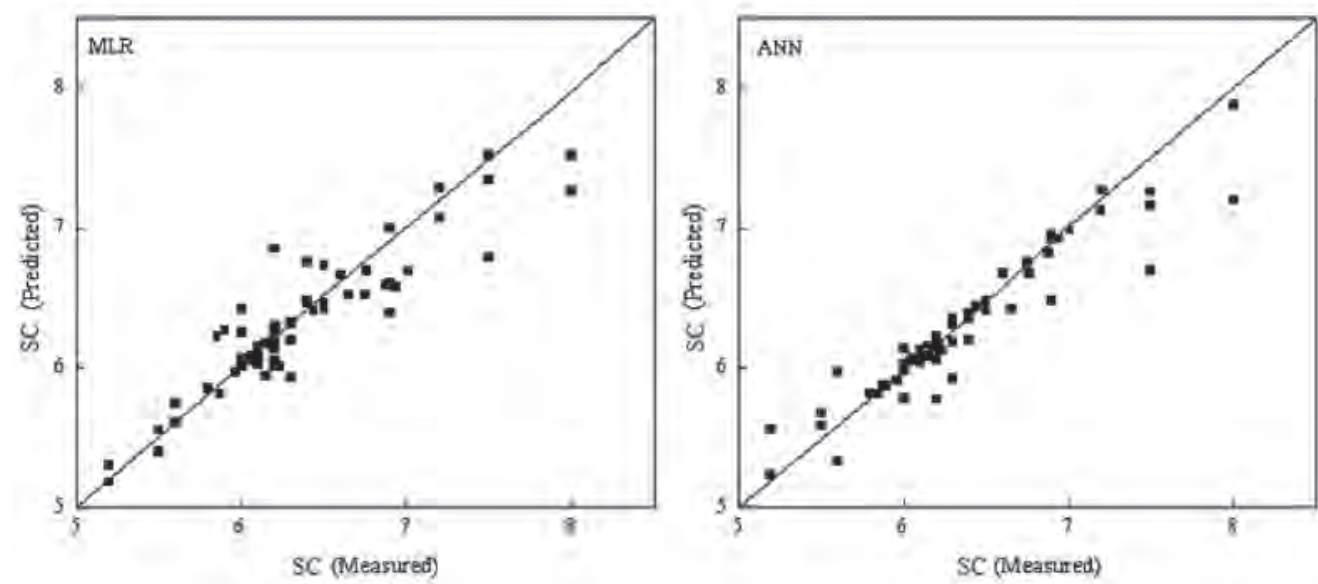

Fig. 5. Comparison of measured $S C$ values with predicted values obtained using ANN and MLR models.

parameters of the network (weights and biases). Generalization ability of the trained ANN was further checked on a test set. The test procedure evaluates the capability of the network to predict new quality indices (maximum $\mathrm{CF}, \mathrm{SC}$ and $\mathrm{pH}$, in this case), not included in the training. The results are shown in Table 4. The correlations of the training model for $\mathrm{CF}, \mathrm{SC}$ and $\mathrm{pH}$ for peaches were as high as $0.96,0.85$ and 0.91 , with SEC values of $0.19,0.21$ and 0.15 , respectively. When the models were used to test samples, prediction results were also high $\left(R^{2}=0.90,0.81,0.87\right)$ : SEP values of $0.53,0.38$ and 0.33 . The ANN models appeared to have a high ability of prediction. 

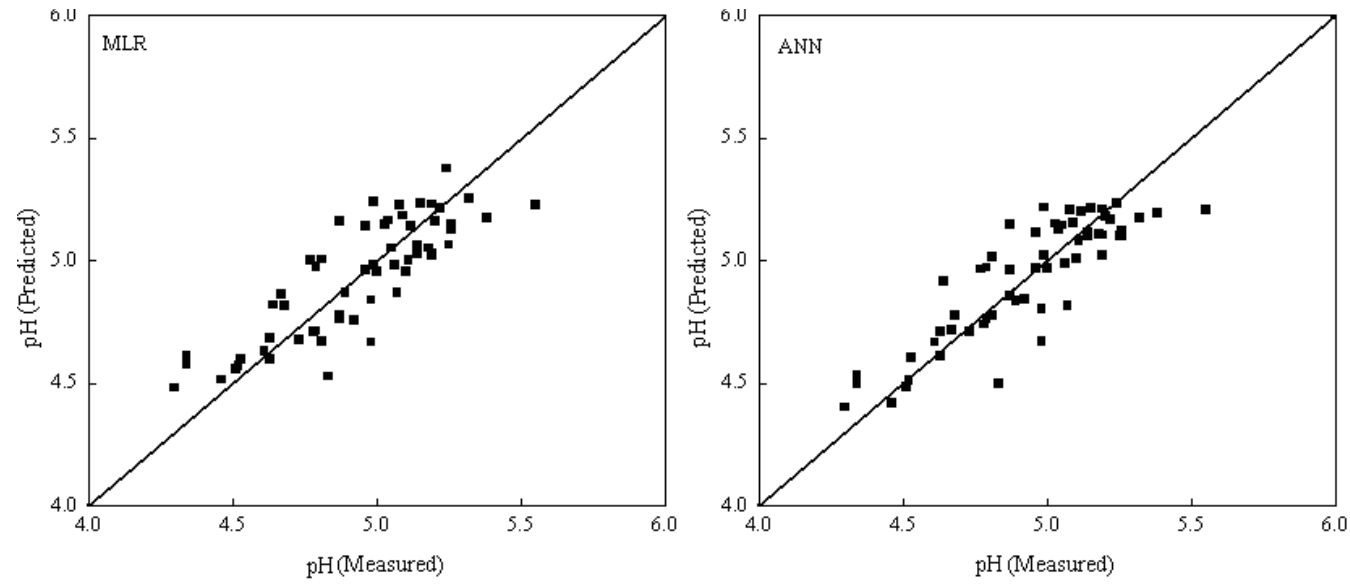

Fig. 6. Comparison of measured $p H$ values with predicted values obtained using ANN and MLR models.

Table 4

Results of prediction of fruit quality indices obtained on the basis of electronic nose signals (ANN model).

\begin{tabular}{ccccccc}
\hline Quality indices & \multicolumn{7}{c}{ ANN } \\
\hline \multicolumn{7}{c}{ Calibration } \\
\hline CF & 0.96 & 0.19 & 2.96 & 0.90 & 0.53 & 6.39 \\
SC & 0.82 & 0.21 & 5.87 & 0.81 & 0.38 & 6.21 \\
pH & 0.91 & 0.15 & 2.22 & 0.87 & 0.33 & 3.13 \\
\hline
\end{tabular}

\subsection{Comparison of ANN and MLR models}

Graphical comparisons of ANN and MLR analyses are shown in Figs. 4-6, where the $\mathrm{CF}, \mathrm{SC}$ and $\mathrm{pH}$ values calculated using the respective models are plotted against the experimental values. Figures 4-6 show the predictive ability of the MLR or ANN model when applied to the test set. Inspection of these plots clearly reveals that the prediction of maturity indices using the neural network is superior to that using MLR. In addition to the correlation coefficient, the overall agreement between observed and predicted values is quantified by the average percentage error (ERR) reported for each data set, as shown in the related table and defined as

$$
E R R=\frac{1}{n} \sum_{i=1}^{n} a b s\left(\frac{t_{i}-y_{i}}{t_{i}}\right) \times 100,
$$


where $n$ is the number of data in a given set, and $t_{i}$ and $y_{i}$ are the measured and predicted values, respectively.

The MLR model is effective for describing the maturity indices of the three selected sets of peaches in training and prediction, as shown by the high correlation coefficients $\left(R^{2}=0.87\right.$ for $\mathrm{CF} ; R^{2}=0.79$ for SC; $R^{2}=0.81$ for $\left.\mathrm{pH}\right)$ and the relatively low ERR $(9.66 \%$, $7.68 \%$ and $3.6 \%$ in the test set, respectively). The feed-forward neural network provides an accurate quality indices model, with a high correlation $\left(R^{2}=0.90,0.81,0.87\right)$ between predicted and measured values and a relatively low ERR $(6.39 \%, 6.21 \%, 3.13 \%)$ for prediction. Although the MLR model provided a quite satisfactory correlation in fitting, prediction was less accurate than the ANN model. $R^{2}$ values and ERR were always slightly higher than those obtained using the ANN model.

\section{Conclusions}

To sum up, the MLR and ANN methods allow the determination of the CF, SC and $\mathrm{pH}$ of peaches using the electronic nose. The superior performance of the analysis carried out using the ANN method has been demonstrated when comparing the correlation coefficient $\left(R^{2}\right)$ and average percent error (ERR) with those found by the MLR method. The results indicate that it is possible to use this nondestructive technique for measuring peach quality characteristics. The MLR and ANN methods have the potential to estimate chemical and physical properties from fruit aroma.

\section{Acknowledgements}

The authors acknowledge the financial support of the Chinese National Foundation of Nature and Science through Project 3057746 and the financial support of the Program for New Century Excellent Talents in Chinese Universities through Project NCET-04-0544.

\section{References}

1 J. Brezmes, E. Llobet, X. Vilanova, G. Saiz and X. Correig: Sens. Actuators, B 69 (2000) 223.

2 L. P. Pathange, P. Mallikarjunan, R. P. Marini, S. Okeefe and D. Vaughan: J. Food Eng. 77 (2006) 1018.

3 E. Molto, E. Selfa, J. Ferriz, E. Conesa and A. Gutierrez: J. Agric. Eng. Res. 72 (1999) 311.

4 C. D Natale, A. Macagnano, E. Martinelli, R. Paolesse, E Proietti and A D'amicro: Sens. Actuators, B 78 (2001) 26.

5 S. Saevels, J. Lammertyn, A. Z. Berna, E. A. Veraverbeke, C. D. Natale and B. M. Nicolai: Postharvest Biol. Technol. 31 (2004) 9.

6 C. Y. Li, P. Heinemann, J. Irudayaraj and D. Peterson: 2005 ASAE Annual International Meeting, Tampa, Florida, USA, July (2005) 17.

7 C. D. Natale, A. Macagnano, E. Martinelli, R. Paolesse, E. Proietti and A. D'amicro: Sens. Actuators, B 77 (2001) 561.

8 C. D. Natale, M. Zude-Sasse, A. Macagnano, R. Paolesse, B. Herold and A. D'Amico: Anal. Chim. Acta 459 (2002) 107. 
9 T. E. San, D. C. Slaughter and J. F. Thompson: Postharvest Biol. Technol. 35 (2005) 177.

10 S. Saevels, J. Lammertyn, A. Z. Berna, E. A. Veraverbeke, C. D. Natale and B. M. Nicolar: Postharvest Biol. Technol. 30 (2003) 3.

11 A. H. Gomez, J. Wang, G. X. Hu and A. G. Pereira: LWT 40 (2007) 681.

12 J. Brezmes, E. Llobet, X. Vilanova, J. Orts, G. Saiz and X. Correig: Sens. Actuators, B 80 (2001) 41.

13 T. Eklöv, G. Johansson, F. Winquist, I. Lundström: J. Sci. Food Agric. 76 (1998) 525.

14 P. N. Jensen, G. Bertelsen and F. V. D. Berg: J. Sci. Food Agric. 85 (2005) 206.

15 J. M. Rodriguez-Nogales: Food Chem. 98 (2006) 782.

16 J. M. Rodriguez-Nogales and F. Vazquez: Food Control 18 (2007) 580.

17 F. Pena, S. Cardenas, M. Gallego and M. Valcarcel: J. Chromatogr., A 1074 (2005) 215.

18 S. N. Jha, S. Chopra and A. R. P. Kingsly: J. Food Eng. 78 (2007) 22.

19 S. N. Jha, A. R. P. Kingsly and S. Chopra1: Biosystems Eng. 94 (2006) 397.

20 A. H. Gomez, Y. He and A. G. Pereira: J. Food Eng. 77 (2006) 313.

21 R. E. Ren and H. W. Wang: Multivariate Statistical Data Analysis-Theory, Method, Example (National Defence Industry Press, Beijing, 1997) pp. 34-119 (in Chinese).

22 H. K. Ekenel and B. Sankur: Pattern Recognition Lett. 25 (2004) 1377.

23 K. Neely, C. Taylor, O Prosser and P. F Hamlyn: Meat Science 58 (2001) 53.

24 H. C. Yu, J. Wang and Y. Xu: Sens. Mater. 19 (2007) 275.

25 Q. Y. Tang and M. G. Feng: DPS Data Processing System for Practical Statistics (Science Press, Beijing, 2002) pp. 294-301 (in Chinese).

26 J. Lozano, J. P. Santos and M. C. Horrillo: Talanta 67 (2005) 610. 